\title{
Lipophilic teicoplanin pseudoaglycon derivatives are active against vancomycin- and teicoplanin-resistant enterococci
}

\author{
Zsolt Szúcss ${ }^{1}$ Ilona Bereczki ${ }^{1}$, Magdolna Csávás ${ }^{1}$, Erzsébet Rőth ${ }^{1}$, Anikó Borbás ${ }^{1}$, Gyula Batta ${ }^{2}$, \\ Eszter Ostorházi ${ }^{3}$, Réka Szatmári ${ }^{3}$ and Pál Herczegh ${ }^{1}$
}

A selection of nine derivatives of teicoplanin pseudoaglycon were tested in vitro against clinical vancomycin-resistant Enterococcus strains possessing vanA, vanB or both genes. The bacteria were characterized by PCR for the identification of their resistance genes. The tested compounds contain lipoic acid, different carbohydrates and aryl groups as lipophilic moieties. About one-third of the teicoplanin-resistant strains were shown to be susceptible to one or more of the glycopeptide derivatives. The Journal of Antibiotics (2017) 70, 664-670; doi:10.1038/ja.2017.2; published online 1 February 2017

\section{INTRODUCTION}

In recent years, the emergence of widespread antibiotic resistance has been getting increasing publicity, while there is also a worldwide attempt to provide financial support for the research and development of new antibiotics. ${ }^{1,2}$ Also, numerous events are organized to raise awareness to this global health-care crisis. Although the discovery of effective antibiotics against resistant Gram-negative bacteria such as Escherichia coli, Klebsiella pneumoniae, Pseudomonas aeruginosa, etc. are of main interest, many resistant Gram-positive bacteria (for example, Clostridium difficile, multidrug-resistant Staphylococcus aureus, vancomycin-resistant enterococci) are also found on the list of the most dangerous organisms causing life-threatening infections, 3,4 therefore constant research in this area should not be neglected.

An enormous collection of derivatives of glycopeptide antibiotics has been synthesized by numerous research groups throughout the years ${ }^{5-12}$ to yield compounds that are active against resistant Gram-positive bacteria, unlike the original molecules. In several cases, the chemical modifications did not only improve the antibacterial spectrum or activity but also many of them came out to be superior to the parent compounds. The successful development and approval of telavancin, ${ }^{13}$ dalbavancin ${ }^{14}$ and oritavancin ${ }^{15}$ proves that semisynthetic work, especially lipophilic derivatization, is undoubtedly useful for preparing compounds with favorable biological characteristics.

Besides others, we have been working on the chemical transformations of glycopeptide antibiotics for a long time. Our synthetic work has essentially been focused on derivatives of the acid hydrolysis products of glycopeptides; that is, aglycons or teicoplanin pseudoaglycon. In order to obtain new derivatives with improved antibacterial activity, in the synthetic work we have carried out in this field, we introduced a large series of more or less lipophilic groups at the
$N$-terminal position of aglycons or pseudoaglycons prepared from ristocetin, teicoplanin or dechloroteicoplanin. ${ }^{16-19}$ Compounds obtained in this way form nanosized aggregates in water. ${ }^{20,21}$ This phenomenon; that is, formation of multivalent clusters, can be one of the explanations of their improved antibacterial activity. In the sense of antibacterial activity, our best results were mostly achieved by starting from the $\mathrm{N}$-acetylglucosaminyl teicoplanin aglycon. The antibacterial activity of these compounds has been evaluated on a standard panel of Gram-positive bacteria, including one vancomycin and one teicoplanin-resistant Enterococcus strain possessing resistance genes $v a n B$ or van $A$ accordingly, but none of the compounds have ever been tested against multiple clinical strains of vancomycinresistant Enterococcus (VRE).

Although, as mentioned, several large systematic series of teicoplanin derivatives are found in the literature, the panel of bacteria for the measurement of bioactivity rarely includes more than a few glycopeptides-resistant Enterococcus strains and only a limited number of derivatives (mainly candidates for further development) are tested against larger collections of VRE. Here we wish to report the antibacterial activity of nine of our most active teicoplanin pseudoaglycon derivatives from recent years' work against a collection of clinical isolates of VRE.

\section{MATERIALS AND METHODS}

\section{General information}

Optical rotations were measured at room temperature with a Perkin-Elmer 241 automatic polarimeter (Perkin-Elmer, Waltham, MA, USA). TLC was performed on Kieselgel $60 \mathrm{~F}_{254}$ (Merck, Darmstadt, Germany) with detection by immersing into $5 \%$ ethanolic sulfuric acid/ammonium molybdate solution followed by heating, or in the case of the teicoplanin derivatives, Pauly's reagent was used for detection. Flash column chromatography was performed on Silica

${ }^{1}$ Department of Pharmaceutical Chemistry, University of Debrecen, Debrecen, Hungary; ${ }^{2}$ Department of Organic Chemistry, University of Debrecen, Debrecen, Hungary and ${ }^{3}$ Department of Medical Microbiology, Semmelweis University, Budapest, Hungary

Correspondence: Professor P Herczegh, Department of Pharmaceutical Chemistry, University of Debrecen, Egyetem tér 1, Debrecen H-4010, Hungary.

E-mail: herczeghp@gmail.com

Dedicated to Prof. Satoshi Ōmura, the winner of the Nobel Prize 2015 in Physiology or Medicine.

Received 19 October 2016; revised 6 December 2016; accepted 18 December 2016; published online 1 February 2017 
gel 60 (Merck $0.040-0.063 \mathrm{~mm}$ ). Organic solutions were dried over $\mathrm{Na}_{2} \mathrm{SO}_{4}$ and concentrated in vacuum. The ${ }^{1} \mathrm{H}$ NMR $(360,400$ and $500 \mathrm{MHz})$ and ${ }^{13} \mathrm{C}$ NMR (91, 101 and $125.76 \mathrm{MHz}$ ) spectra were recorded with Bruker DRX-360, DRX-400 and Bruker Avance II 500 spectrometers at $25^{\circ} \mathrm{C}$. Chemical shifts are referenced to $\mathrm{Me}_{4} \mathrm{Si}\left(0.00\right.$ p.p.m. for $\left.{ }^{1} \mathrm{H}\right)$ and to the solvent signals (dimethylsulfoxide (DMSO) $-\mathrm{d}_{6}: 2.50$ p.p.m. for ${ }^{1} \mathrm{H}$ and $\mathrm{CDCl}_{3}$ : 77.16 p.p.m. DMSO- $\mathrm{d}_{6}: 39.52$ p.p.m. for ${ }^{13} \mathrm{C}$ ). Matrix assisted laser desorption/ionizationtime of flight MS (MALDI-TOF-MS) analyses of the compounds were carried out in the positive reflectron mode using a BIFLEX III mass spectrometer (Bruker, Bremen, Germany) equipped with delayed-ion extraction. 2,5-Dihydroxybenzoic acid was used as matrix and $\mathrm{CF}_{3} \mathrm{COONa}$ as cationizing agent in dimethylformamide (DMF). ESI-TOF MS spectra were recorded by a microTOF-Q type QqTOFMS mass spectrometer (Bruker) in the positive ion mode using $\mathrm{MeOH}$ as the solvent. Elemental analysis was performed on an Elementar Vario MicroCube instrument. The $\log P$ values were calculated using the $\log P$ calculation plugin of Marvin Sketch (version 16.5.2) from ChemAxon (Budapest, Hungary) using the Consensus Method with electrolyte concentrations of $0.1 \mathrm{M}$.

\section{Bacterial strains}

The Department of Medical Microbiology, Semmelweis University, Budapest, Hungary cultured and characterized 44 VRE strains. All the strains were isolated from routine laboratory samples of patients suffering from wound, urinary tract or blood stream infections. Clinical samples were cultured on COS agar (Columbia agar $+5 \%$ sheep blood, Biomérieux, Budapest, Hungary), and vancomycin-resistant strains were isolated on VRE chromogenic medium (Biomérieux). Species of strains were identified by MALDI-TOF-MS. Minimum inhibitory concentrations (MICs; $\mathrm{mg} \mathrm{l}^{-1}$ ) were determined for vancomycin and teicoplanin on Mueller Hinton E agar (Biomérieux using MIC strip tests (Liofilchem, Roseto degli Abruzzi, Italy), according to the manufacturer's instructions, and by using a direct colony suspension equivalent to a McFarland standard of 0.5 . Testing conditions also included incubation at $35.5^{\circ} \mathrm{C}$ for $24 \mathrm{~h}$. All results were interpreted by using breakpoints for susceptibility and resistance according to the European Committee on Antimicrobial Susceptibility Testing (EUCAST) 22 Concerning vancomycin/ teicoplanin MIC breakpoints, strains with an MIC> 4 or $2 \mathrm{mgl}^{-1}$ were considered to be vancomycin- or teicoplanin-resistant, respectively.

\section{MIC of lipophilic teicoplanin pseudoaglycon derivatives}

The efficacy of the prepared compounds was determined with the broth microdilution method according to the EUCAST guideline. Bacterial strains were grown on $\mathrm{COS}$ agar at $35.5^{\circ} \mathrm{C}$ overnight. Appropriate numbers of colonies were suspended in physiological saline in order to reach the density of 0.5 McFarland for inoculation.

Stock solutions containing the substances were prepared in distilled water and DMSO (1:1). These were two-fold serially diluted from 20 to $0.044 \mathrm{mg} \mathrm{l}^{-1}$ in cation-adjusted Mueller-Hinton broth (Biolab, Budapest, Hungary) and $100 \mu \mathrm{l}$ of each dilution was transferred into microplate holes. Inoculation was carried out with $10 \mu \mathrm{l}$ of each bacterial suspension. Incubation was performed at $35^{\circ} \mathrm{C}$ for $24 \mathrm{~h}$ and determination of MIC was made with the naked eyes on a black background.

\section{Determination of $v a n A$ and $v a n B$ genes}

Enterococcus strains were retrieved from storage at $-80^{\circ} \mathrm{C}$ on Cryobank breads (Mast Diagnostica, Reinfeld, Germany), cultured on COS agar and incubated overnight at $35.5^{\circ} \mathrm{C}$. A turbid suspension of the subculture was made in PCR water and DNA was isolated with the GeneAll Ribospin Extraction Kit (Geneall Biotechnology, Seoul, Korea).

The presence of glycopeptides-resistance genes was investigated by PCR using primers for the identification of $\operatorname{van} A$ or $\operatorname{van} B$ sequence described by Dutka-Malen et al. ${ }^{23}$ The full reaction mixture $(25 \mu \mathrm{l})$ included $12.5 \mu \mathrm{l}$ master mix (ImmoMix Red, BIOLINE, London, UK), $0.5 \mu \mathrm{l}$ forward primer, $0.5 \mu \mathrm{l}$ reverse primer, $6.5 \mu \mathrm{l}$ PCR water and $5 \mu \mathrm{l}$ DNA template. PCR thermal profile was $94{ }^{\circ} \mathrm{C} 2 \mathrm{~min},\left(92{ }^{\circ} \mathrm{C} 1 \mathrm{~min}, 54{ }^{\circ} \mathrm{C} 1 \mathrm{~min}, 72{ }^{\circ} \mathrm{C} 1 \mathrm{~min}\right) 30$ cycles, $72{ }^{\circ} \mathrm{C}$ $10 \mathrm{~min}$. PCR products were detected by Gelgreen nucleic acid gel stain (BIOLINE) in $2 \%$ agarose under UV light.

\section{Compound 1}

Teicoplanin pseudoaglycon 10 (200 mg, $0.14 \mathrm{mmol})$ was dissolved in dry DMF $(3 \mathrm{ml})$ and $\mathrm{Et}_{3} \mathrm{~N}(20 \mu \mathrm{l})$ and compound $11^{24}(57.6 \mathrm{mg}, 0.19 \mathrm{mmol})$ was added. After $3 \mathrm{~h}$, TLC showed complete disappearance of starting material 10. The reaction mixture was concentrated and the residue was purified by flash column chromatography (toluene:MeOH $4: 6+1 \%$ acetic acid) to give 1 $(153 \mathrm{mg}, 68 \%)$ as an off-white powder. MALDI-TOF-MS $\mathrm{m} / \mathrm{z} 1611.35$ $(\mathrm{M}+\mathrm{Na})^{+}$; calcd. 1611.32 for $\mathrm{C}_{74} \mathrm{H}_{70} \mathrm{Cl}_{2} \mathrm{~N}_{8} \mathrm{NaO}_{24} \mathrm{~S}_{2}$.

\section{Compound 2}

Teicoplanin pseudoaglycon 10 (200 mg, $0.14 \mathrm{mmol})$ was dissolved in dry DMF $(3 \mathrm{ml})$ and $\mathrm{Et}_{3} \mathrm{~N}(20 \mu \mathrm{l}, 0.14 \mathrm{mmol})$ and compound $12^{24}(77.2 \mathrm{mg}, 0.19 \mathrm{mmol})$ was added. After $3 \mathrm{~h}$, TLC showed complete disappearance of starting material 10. The reaction mixture was concentrated and the residue was purified by flash column chromatography (toluene:MeOH $1: 1+1 \%$ acetic acid) to give $2(166 \mathrm{mg}, 68 \%)$ as an off-white powder. MALDI-TOF-MS $\mathrm{m} / \mathrm{z} 1724.45$ $(\mathrm{M}+\mathrm{Na})^{+}$; calcd. 1724.41 for $\mathrm{C}_{80} \mathrm{H}_{81} \mathrm{Cl}_{2} \mathrm{~N}_{9} \mathrm{NaO}_{25} \mathrm{~S}_{2}$.

\section{Methyl 2,3-di-O-n-butyl-4,6-O-p-methoxybenzylidene- $\alpha$-D-gluco- pyranoside (14)}

To the mixture of $\mathrm{NaH}$ (50\% in mineral oil, $922 \mathrm{mg}, 19.2 \mathrm{mmol}$ ) washed with hexane and dry DMF $(30 \mathrm{ml})$, methyl 4,6-O-p-methoxybenzylidene- $\alpha$-Dglucopyranoside $13^{25}$ (1.5 g, $4.8 \mathrm{mmol}$ ) was added, and the suspension was stirred for $45 \mathrm{~min}$ under argon. Then $n$-butyl bromide $(1.55 \mathrm{ml}, 14.4 \mathrm{mmol})$ was added in four portions in an hour. After two days, $\mathrm{MeOH}(2 \mathrm{ml})$ and distilled water $(50 \mathrm{ml})$ was added carefully, and the mixture was stirred for half an hour. The precipitated solids were filtered off and purified by flash chromatography ( $n$-hexane:acetone 9:1) to give $14(1.30 \mathrm{~g}, 64 \%)$ as a white powder. $[\alpha]_{\mathrm{D}}^{25}+46.7\left(\mathrm{c} 0.08, \mathrm{CHCl}_{3}\right)$ MALDI-TOF-MS $\mathrm{m} / \mathrm{z} 447.36(\mathrm{M}+\mathrm{Na})^{+}$; calcd. 447.24 for $\mathrm{C}_{23} \mathrm{H}_{36} \mathrm{NaO}_{7} .{ }^{1} \mathrm{H}$ NMR $\left(400 \mathrm{MHz}, \mathrm{CDCl}_{3}\right) \delta$ (p.p.m.): 7.43 (d, $J=8.7 \mathrm{~Hz}, 2 \mathrm{H}$, aromatic), 6.90 (d, $2 \mathrm{H}, J=8.7 \mathrm{~Hz}$ aromatic), $5.51(\mathrm{~s}, 1 \mathrm{H}, \mathrm{H}$ acetalic), $4.81(\mathrm{~d}, J=3.6 \mathrm{~Hz}, 1 \mathrm{H}, \mathrm{H}-1), 4.27$ (dd, $J=9.8,4.5 \mathrm{~Hz}, 1 \mathrm{H}, \mathrm{CH}_{2}$ ), $3.85-3.60(\mathrm{~m}, 10 \mathrm{H}), 3.50(\mathrm{t}, J=9.3 \mathrm{~Hz}, 1 \mathrm{H}), 3.45$ (s, 3H, OMe), 3.37 (dd, $J=9.3,3.7 \mathrm{~Hz}, 1 \mathrm{H}, \mathrm{H}-2), 1.63-1.52\left(\mathrm{~m}, 4 \mathrm{H}\right.$, butyl $\left.\mathrm{CH}_{2}\right), 1.46-1.33$ (m, $4 \mathrm{H}$, butyl $\left.\mathrm{CH}_{2}\right), 0.94\left(\mathrm{t}, J=7.4 \mathrm{~Hz}, 3 \mathrm{H}\right.$, butyl $\left.\mathrm{CH}_{3}\right), 0.89(\mathrm{t}, J=7.4 \mathrm{~Hz}, 3 \mathrm{H}$, butyl $\left.\mathrm{CH}_{3}\right) .{ }^{13} \mathrm{C} \mathrm{NMR}\left(101 \mathrm{MHz}, \mathrm{CDCl}_{3}\right) \delta$ (p.p.m.): 160.3, 130.0, 127.3, 113.5 (6C, aromatic), 101.2 (1C, acetalic), 99.1 (1C, C-1), 82.0, 80.4, 78.3 (3C, skeleton), 73.4, 72.0 (2C, butyl $\mathrm{CH}_{2}$ ), 69.0 (1C, C-6), 62.4 (1C, C-5), 55.2 (2C, $2 \mathrm{OMe}$ ), 32.3, 32.1, 19.2, 19.1 (4C, butyl $\mathrm{CH}_{2}$ ), 13.8 (2C, butyl $\mathrm{CH}_{3}$ ).

\section{Methyl 2,3-di-O-n-butyl-4-O-p-methoxybenzyl- $\alpha$-D- glucopyranoside (15)}

p-Methoxybenzylidene derivative $\mathbf{1 4}(1.20 \mathrm{~g}, 2.24 \mathrm{mmol})$ was dissolved in a mixture of dry $\mathrm{CH}_{2} \mathrm{Cl}_{2}(20 \mathrm{ml})$ and dry $\mathrm{Et}_{2} \mathrm{O}(7 \mathrm{ml})$ under an argon atmosphere. The stirred mixture was cooled to $0{ }^{\circ} \mathrm{C}$, and $\mathrm{LiAlH}_{4}(382 \mathrm{mg}, 10.1 \mathrm{mmol})$ was added in four portions. Dry $\mathrm{Et}_{2} \mathrm{O}(7 \mathrm{ml})$ was cooled to $0{ }^{\circ} \mathrm{C}$ by the use of drying tube filled with $\mathrm{CaCl}_{2}$, and $\mathrm{AlCl}_{3}(447 \mathrm{mg}, 3.4 \mathrm{mmol}$ ) was added. The solution of $\mathrm{AlCl}_{3}$ was stirred for $5 \mathrm{~min}$, then it was added to the previously prepared mixture containing the $p$-methoxybenzylidene derivative and it was stirred at $0{ }^{\circ} \mathrm{C}$ under an argon atmosphere. After $1 \mathrm{~h}$, ethyl acetate $(45 \mathrm{ml})$ was added to the reaction mixture and it was stirred for $10 \mathrm{~min}$, then water $(10 \mathrm{ml})$ was added, and the mixture was stirred for further $10 \mathrm{~min}$. The mixture was filtered through a Celite pad (Sigma Aldrich, Budapest, Hungary), and the solid part was washed with ethyl acetate. The filtrate was diluted with ethyl acetate $(100 \mathrm{ml})$ and washed twice with water $(2 \times 40 \mathrm{ml})$. The organic solution was dried and concentrated. The residue was purified by flash column chromatography ( $n$-hexane:acetone 8:2) to yield $15(902 \mathrm{mg}, 75 \%)$ as a colorless syrup. $[\alpha]_{\mathrm{D}}{ }^{25}+78.43$ (c 0.07 , $\left.\mathrm{CHCl}_{3}\right)$; MALDI-TOF-MS $m / z 449.36(\mathrm{M}+\mathrm{Na})^{+}$; calcd. 449.25 for $\mathrm{C}_{23} \mathrm{H}_{38} \mathrm{NaO}_{7}$. ${ }^{1} \mathrm{H} \mathrm{NMR}\left(400 \mathrm{MHz}, \mathrm{CDCl}_{3}\right) \delta$ (p.p.m.): 7.29 (d, $J=8.8 \mathrm{~Hz}, 2 \mathrm{H}$, aromatic), 6.90 (d, $J=8.6 \mathrm{~Hz}, 2 \mathrm{H}$, aromatic), $4.85\left(\mathrm{~d}, J=10.7 \mathrm{~Hz}, 1 \mathrm{H}\right.$, benzyl $\left.\mathrm{CH}_{2}\right), 4.78$ (d, $J=3.5 \mathrm{~Hz}, 1 \mathrm{H}, \mathrm{H}-1), 4.60\left(\mathrm{~d}, J=10.7 \mathrm{~Hz}, 1 \mathrm{H}\right.$, benzyl $\left.\mathrm{CH}_{2}\right), 3.94-3.86(\mathrm{~m}$, $1 \mathrm{H}) ; 3.82(\mathrm{~s}, 3 \mathrm{H}, \mathrm{OMe}), 3.81-3.57(\mathrm{~m}, 4 \mathrm{H}), 3.46-3.44(\mathrm{~m}, 1 \mathrm{H}), 3.40(\mathrm{~s}, 3 \mathrm{H}$, OMe), $3.30(\mathrm{dd}, J=9.6,3.6 \mathrm{~Hz}, 1 \mathrm{H}, \mathrm{H}-2), 1.67-1.57\left(\mathrm{~m}, 4 \mathrm{H}\right.$, butyl $\mathrm{CH}_{2}$ ), 1.48-1.36 (m, $4 \mathrm{H}$, butyl $\left.\mathrm{CH}_{2}\right), 0.95\left(\mathrm{t}, 6 \mathrm{H}, J=7.4 \mathrm{~Hz}\right.$, butyl $\left.\mathrm{CH}_{3}\right) .{ }^{13} \mathrm{C} \mathrm{NMR}$ $\left(101 \mathrm{MHz}, \mathrm{CDCl}_{3}\right) \delta$ (p.p.m.): 159.3, 130.4, 129.8, 113.9 (6C, aromatic), 98.0 (1C, C-1), 81.6, 80.9, 77.3 (3C, skeleton), 74.6 (1C, benzyl $\mathrm{CH}_{2}$ ), 73.4, 71.4 
(2C, butyl $\mathrm{CH}_{2}$ ), 70.6 (1C, C-5), 62.0 (1C, C-6), 55.2, 55.1 (2C, OMe), 32.7, 32.1, 19.4, 19.1 (4C, butyl $\mathrm{CH}_{2}$ ), 14.1, 14.0 (2C, butyl $\mathrm{CH}_{3}$ ).

Methyl 2,3-di-O-n-butyl-4-O-p-methoxybenzyl-6-O-(2,5,8,11, 14-pentaoxaheptadec-16-ynyl)- $\alpha$-D-glucopyranoside (16)

To the solution of sugar derivative $15(500 \mathrm{mg}, 1.17 \mathrm{mmol})$ in dry DMF $(20 \mathrm{ml}), \mathrm{NaH}$ was added $(50 \%$ in mineral oil, $112 \mathrm{mg}, 2.34 \mathrm{mmol})$, and the mixture was stirred for $20 \mathrm{~min}$ under an argon atmosphere. The solution of triethylene glycol 2-bromoethyl propargyl ether ${ }^{26}(414 \mathrm{mg}, 1.40 \mathrm{mmol})$ in dry DMF $(2 \mathrm{ml})$ was added to the mixture and it was stirred overnight. Additional $\mathrm{NaH}(50 \%, 50 \mathrm{mg})$ and triethylene glycol 2-bromoethyl propargyl ether $(50 \mathrm{mg})$ were added to the reaction mixture, and it was stirred at $60^{\circ} \mathrm{C}$ for 2 days. The reaction was quenched by the addition of $\mathrm{MeOH}(2 \mathrm{ml})$ and water $(2 \mathrm{ml})$ and the mixture was stirred for $10 \mathrm{~min}$. After removing the solvents under vacuum, the residue was purified by flash chromatography ( $n$-hexane: acetone $95: 5)$ to yield $16(360 \mathrm{mg}, 48 \%)$ as a colorless syrup. $[\alpha]_{\mathrm{D}}{ }^{25}+63.40$ (c 1.4, $\left.\mathrm{CHCl}_{3}\right)$ ESI-TOF-MS $\mathrm{m} / z 663.3728(\mathrm{M}+\mathrm{Na})^{+}$; calcd. 663.3715 for $\mathrm{C}_{34} \mathrm{H}_{56} \mathrm{NaO}_{11} .{ }^{1} \mathrm{H}$ NMR (400 MHz, $\left.\mathrm{CDCl}_{3}\right) \delta$ (p.p.m.) 7.31-7.23 (m, $2 \mathrm{H}$, aromatic); $6.90-6.84\left(\mathrm{~m}, 2 \mathrm{H}\right.$, aromatic); $4.83-4.75\left(\mathrm{~m}, 2 \mathrm{H}\right.$, benzyl $\mathrm{CH}_{2}$ and $\mathrm{H}-1) ; 4.57\left(\mathrm{~d}, J=10.5 \mathrm{~Hz}, 1 \mathrm{H}\right.$, benzyl $\left.\mathrm{CH}_{2}\right) ; 4.19(\mathrm{~d}, J=2.3 \mathrm{~Hz}, 2 \mathrm{H}$, propargyl $\left.\mathrm{CH}_{2}\right) ; 3.89-3.81(\mathrm{~m}, 1 \mathrm{H}) ; 3.80(\mathrm{~s}, 3 \mathrm{H}, \mathrm{OMe}) ; 3.76-3.53(\mathrm{~m}, 23 \mathrm{H}) ; 3.49$ $(\mathrm{t}, J=9.2 \mathrm{~Hz}, 1 \mathrm{H}) ; 3.38(\mathrm{~s}, 3 \mathrm{H}, \mathrm{OMe}) ; 3.30(\mathrm{dd}, J=9.7,3.5 \mathrm{~Hz}, 1 \mathrm{H}) ; 2.43$ $(\mathrm{t}, J=2.3 \mathrm{~Hz}, 1 \mathrm{H}$, propargyl $\mathrm{CH}) ; 1.67-1.52\left(\mathrm{~m}, 4 \mathrm{H}\right.$, butyl $\left.\mathrm{CH}_{2}\right) ; 1.46-1.32$ (m, 4H, butyl $\left.\mathrm{CH}_{2}\right) ; 0.92-0.83\left(\mathrm{~m}, 6 \mathrm{H}\right.$, butyl $\left.\mathrm{CH}_{3}\right) \cdot{ }^{13} \mathrm{C} \mathrm{NMR}(101 \mathrm{MHz}$, $\left.\mathrm{CDCl}_{3}\right) \delta$ (p.p.m.) 159.2, 130.7, 129.5, 113.7 (6C, aromatic), 98.0 (1C, C-1), 81.6, 80.7, 77.3, 70.0 (4C, skeleton), 74.6, 74.4, 73.3, 71.2, 70.8, 70.7, 70.6, 70.5, 70.4, 70.0, 69.8 (11C, $\mathrm{OCH}_{2}$ tetraethyleneglycol, $\mathrm{OCH}_{2}$ butyl, $\mathrm{OCH}_{2} \mathrm{PMB}$ (p-methoxybenzyl)), 58.3 (1C, C-6), 55.2, 55.0 (2C, OMe), 32.63, 32.02, 19.33, 19.05, (4C, $\mathrm{CH}_{2}$ butyl), 14.0, 13.8 (2C, $\mathrm{CH}_{3}$ butyl).

\section{Methyl 2,3-di-O-n-butyl-6-O-(2,5,8,11,14-pentaoxaheptadec-16- ynyl)- $\alpha$-D-glucopyranoside (17)}

Compound 16 (225 mg, $0.35 \mathrm{mmol}$ ) and DDQ (2,3-dichloro-5,6-dicyano-1, 4-benzoquinone; $120 \mathrm{mg}, 0.53 \mathrm{mmol}$ ) were dissolved in $\mathrm{CH}_{2} \mathrm{Cl}_{2}$ :water 9:1
$(25 \mathrm{ml})$, and the mixture was stirred vigorously. After $1 \mathrm{~h}$, the reaction mixture was diluted with $\mathrm{CH}_{2} \mathrm{Cl}_{2}(300 \mathrm{ml})$, washed with saturated aq $\mathrm{NaHCO}_{3}$ $(2 \times 50 \mathrm{ml})$ and with water $(50 \mathrm{ml})$. The organic phase was dried and concentrated. The residue was purified by flash column chromatography to yield $17(153 \mathrm{mg}, 84 \%)$ as a colorless syrup. $[\alpha]_{\mathrm{D}}{ }^{25}+48.26\left(\mathrm{c} 0.09, \mathrm{CHCl}_{3}\right)$; MALDI-TOF-MS $m / z 655.45(\mathrm{M}+\mathrm{Na})^{+}$; calcd. 655.44 for $\mathrm{C}_{34} \mathrm{H}_{64} \mathrm{NaO}_{10}$. ${ }^{1} \mathrm{H}$ NMR $\left(360 \mathrm{MHz}, \mathrm{CDCl}_{3}\right) \delta$ (p.p.m.): $4.79(\mathrm{~d}, J=3.5 \mathrm{~Hz}, 1 \mathrm{H}, \mathrm{H}-1), 4.21$ (d, $J=2.4 \mathrm{~Hz}, 2 \mathrm{H}$, propargyl $\left.\mathrm{CH}_{2}\right), 3.91-3.82(\mathrm{~m}, 1 \mathrm{H}), 3.80-3.48(\mathrm{~m}, 25 \mathrm{H})$; $3.41(\mathrm{~s}, 3 \mathrm{H}, \mathrm{OMe}), 3.29(\mathrm{dd}, J=9.1,3.5 \mathrm{~Hz}, 1 \mathrm{H}, \mathrm{H}-2), 2.45(\mathrm{t}, J=2.4 \mathrm{~Hz}, 1 \mathrm{H}$, propargyl $\mathrm{CH}), 1.62-1.51\left(\mathrm{~m}, 4 \mathrm{H}\right.$, butyl $\left.\mathrm{CH}_{2}\right), 1.43-1.32\left(\mathrm{~m}, 8 \mathrm{H}\right.$, butyl $\left.\mathrm{CH}_{2}\right)$, $0.91\left(\mathrm{t}, J=7.4 \mathrm{~Hz}, 6 \mathrm{H}, \mathrm{CH}_{3}\right) .{ }^{13} \mathrm{C}$ NMR $\left(91 \mathrm{MHz}, \mathrm{CDCl}_{3}\right) \delta$ (p.p.m.): 98.0 (1C, C-1), 80.9, 80.3, 70.0 (3C, skeleton) 74.4, 73.1, 70.9, 70.7, 70.3, 70.0 $\left(\mathrm{OCH}_{2}\right), 68.9$ (1C, C-6), 58.2, 55.0 (1C, OMe), 32.3, 31.9, 19.1, 19.0 (4C, $\mathrm{CH}_{2}$ butyl), 13.8, 13.7 (2C, $\mathrm{CH}_{3}$ butyl).

\section{Compound 4}

To the solution of azido teicoplanin pseudoaglycon $18^{20}(150 \mathrm{mg}, 0.11 \mathrm{mmol})$ and the propargyl derivative $16(81 \mathrm{mg}, 0.126 \mathrm{mmol})$ in dry DMF $(5 \mathrm{ml})$, $\mathrm{CuI}(2 \mathrm{mg}, 0.011 \mathrm{mmol})$ and $\mathrm{Et}_{3} \mathrm{~N}(0.11 \mathrm{mmol}, 15 \mu \mathrm{l})$ were added under an argon atmosphere and the mixture was stirred for $20 \mathrm{~h}$. The mixture was concentrated and the residue was purified by flash column chromatography (toluene: $\mathrm{MeOH} 6: 4+0.1 \%$ acetic acid) to yield $4(84 \mathrm{mg}, 39 \%)$ as a white powder. MALDI-TOF-MS $\mathrm{m} / \mathrm{z} 2089.66(\mathrm{M}+\mathrm{Na})^{+}$; calcd. 2089.66 for $\mathrm{C}_{100} \mathrm{H}_{112} \mathrm{Cl}_{2} \mathrm{~N}_{10} \mathrm{NaO}_{34}$.

\section{Compound 5}

To the solution of azido teicoplanin pseudoaglycon $18(150 \mathrm{mg}, 0.11 \mathrm{mmol})$ and the propargyl derivative $17(66 \mathrm{mg}, 0.126 \mathrm{mmol})$ in dry DMF $(5 \mathrm{ml})$, $\mathrm{CuI}(2 \mathrm{mg}, 0.01 \mathrm{mmol})$ and $\mathrm{Et}_{3} \mathrm{~N}(0.11 \mathrm{mmol}, 15 \mu \mathrm{l})$ were added under an argon atmosphere and the mixture was stirred for 2 days and at $60{ }^{\circ} \mathrm{C}$ for a day. The mixture was concentrated and the residue was purified by flash chromatography (toluene: $\mathrm{MeOH} 6: 4+0.1 \%$ acetic acid) to yield $5(98 \mathrm{mg}$, $48 \%)$ as a white powder. MALDI-TOF-MS $\mathrm{m} / \mathrm{z} 1969.60(\mathrm{M}+\mathrm{Na})^{+}$; calcd. 1969.60 for $\mathrm{C}_{92} \mathrm{H}_{104} \mathrm{Cl}_{2} \mathrm{~N}_{10} \mathrm{NaO}_{33}$.

Table 1 Structure and lipophilicity of teicoplanin pseudoaglycon derivatives 1-9

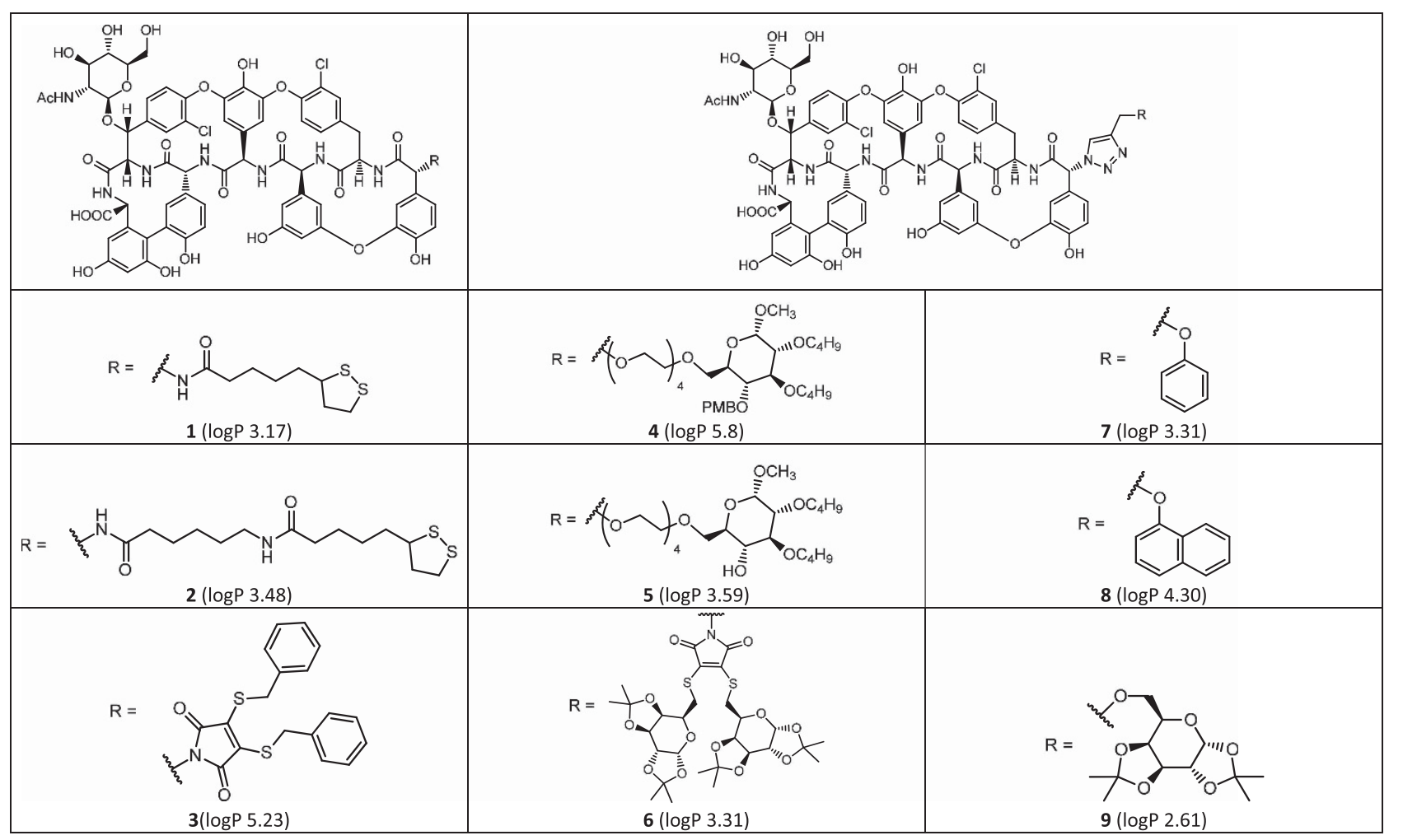


Glycopeptide activities against clinical enterococci

Z Szücs et al

667

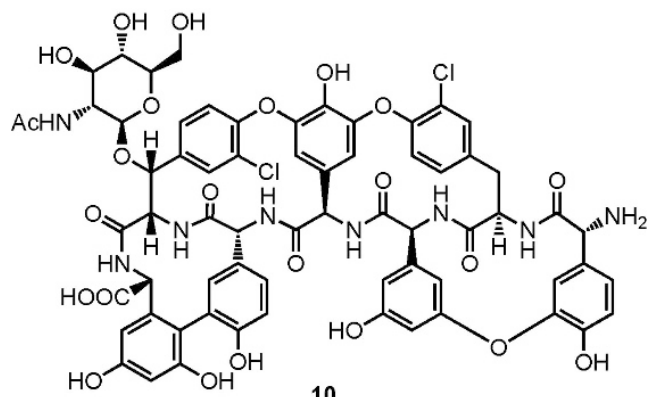<smiles>CC(C)(C)ON1C(=O)CCC1=O</smiles>

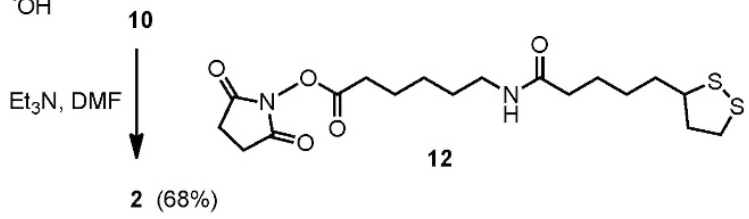

Scheme 1 Conjugation of lipoic acid derivatives 11 and $\mathbf{1 2}$ to the $\mathrm{N}$-terminal part of teicoplanin pseudoaglycon.

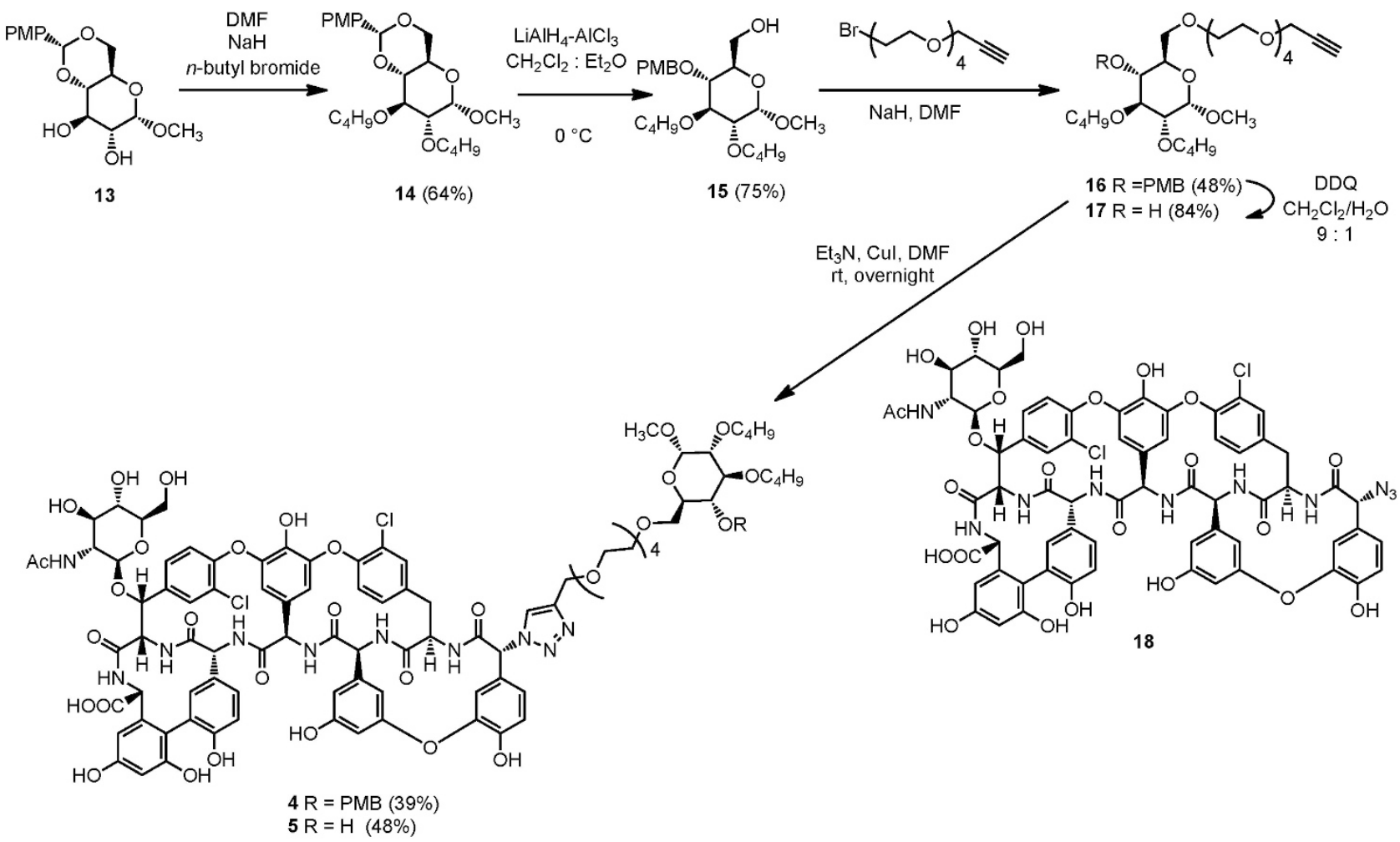

Scheme 2 Derivatization of azide teicoplanin pseudoaglycon with sugar-based lipophilic moieties by CuAAC.

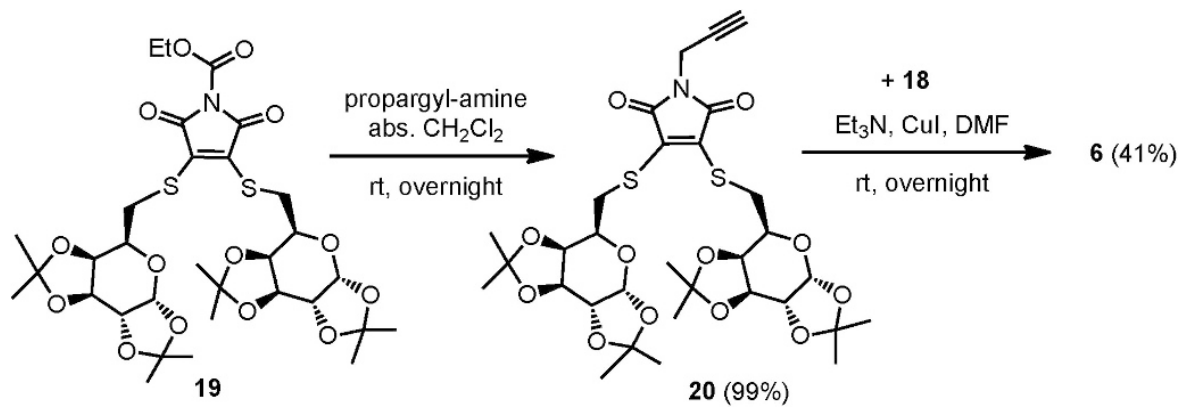

Scheme 3 Coupling of bis-(diisopropylidene- $\alpha$-D-Galp-6-thio)-maleimide $20-18$ by CuAAC.

The Journal of Antibiotics 
Table 2 MIC of compounds $1-9$ against VanB enterococci in $\mu \mathrm{g} \mathrm{ml}^{-1}$

\begin{tabular}{|c|c|c|c|c|c|c|c|c|c|c|c|c|c|}
\hline Number & Strain & $P C R$ & Vancomycin & Teicoplanin & 1 & 2 & 3 & 4 & 5 & 6 & 7 & 8 & 9 \\
\hline 2 & E. faecalis $312 / 2014$ & $\operatorname{van} B$ & 48 & 1.5 & 0.625 & 10 & 0.625 & 2.5 & 5 & 1.25 & 2.5 & 1.25 & 20 \\
\hline 4 & E. faecium 1256/2014 & $\operatorname{van} B$ & 48 & 1 & 1.25 & 5 & 1.25 & 1.25 & 2.5 & 5 & 1.25 & 0.3125 & 10 \\
\hline 5 & E. faecium $3025 / 2014$ & $\operatorname{van} B$ & 12 & 2 & 1.25 & 5 & 1.25 & 1.25 & 5 & 1.25 & 0.625 & 0.625 & 5 \\
\hline 6 & E. faecalis $4035 / 2014$ & $\operatorname{van} B$ & 48 & 2 & 2.5 & 20 & 0.625 & 2.5 & 10 & 5 & 1.25 & 1.25 & 10 \\
\hline
\end{tabular}

Abbreviation: MIC, minimum inhibitory concentration.

Table 3 MIC of compounds 1-9 against VanA enterococci in $\mu \mathrm{g} \mathrm{ml}^{-1}$

\begin{tabular}{|c|c|c|c|c|c|c|c|c|c|c|c|c|c|}
\hline Number & Strain & $P C R$ & Vancomycin & Teicoplanin & 1 & 2 & 3 & 4 & 5 & 6 & 7 & 8 & 9 \\
\hline 9 & E. faecium $276 / 2014$ & $\operatorname{van} A$ & $>96$ & 96 & $>20$ & $>20$ & $>20$ & $>20$ & $>20$ & $>20$ & $>20$ & $>20$ & $>20$ \\
\hline 10 & E. faecium 298/2014 & $\operatorname{van} A$ & $>96$ & 12 & 2.5 & 20 & 5 & 5 & 20 & 20 & 5 & 1.25 & 20 \\
\hline 11 & E. faecalis $675 / 2014$ & $\operatorname{van} A$ & $>96$ & $>96$ & 10 & $>20$ & 2.5 & 20 & $>20$ & 5 & 2.5 & 0.3125 & $>20$ \\
\hline 12 & E. faecalis $863 / 2014$ & $\operatorname{van} A$ & $>96$ & 32 & $>20$ & $>20$ & $>20$ & $>20$ & $>20$ & $>20$ & $>20$ & $>20$ & $>20$ \\
\hline 13 & E. faecalis $1022 / 2014$ & $\operatorname{van} A$ & $>96$ & $>96$ & $>20$ & $>20$ & 10 & 10 & $>20$ & 5 & 10 & 10 & $>20$ \\
\hline 14 & E. faecium 1057/2014 & $\operatorname{van} A$ & $>96$ & $>96$ & $>20$ & $>20$ & $>20$ & $>20$ & $>20$ & $>20$ & $>20$ & $>20$ & $>20$ \\
\hline 15 & E. faecium 1788/2014 & $\operatorname{van} A$ & $>96$ & $>96$ & $>20$ & $>20$ & $>20$ & $>20$ & $>20$ & $>20$ & $>20$ & $>20$ & $>20$ \\
\hline 16 & E. faecium $1876 / 2014$ & $\operatorname{van} A$ & $>96$ & $>96$ & $>20$ & $>20$ & $>20$ & $>20$ & $>20$ & $>20$ & $>20$ & $>20$ & $>20$ \\
\hline 17 & E. faecium 1997/2014 & $\operatorname{van} A$ & $>96$ & $>96$ & $>20$ & $>20$ & $>20$ & $>20$ & $>20$ & $>20$ & $>20$ & $>20$ & $>20$ \\
\hline 18 & E. faecium 2034/2014 & $\operatorname{van} A$ & $>96$ & $>96$ & $>20$ & $>20$ & $>20$ & $>20$ & $>20$ & $>20$ & $>20$ & $>20$ & $>20$ \\
\hline 19 & E. faecium 2076/2014 & $\operatorname{van} A$ & $>96$ & $>96$ & $>20$ & $>20$ & $>20$ & $>20$ & $>20$ & $>20$ & $>20$ & $>20$ & $>20$ \\
\hline 20 & E. faecium 3016/2014 & $\operatorname{van} A$ & $>96$ & 96 & $>20$ & $>20$ & $>20$ & $>20$ & $>20$ & $>20$ & $>20$ & $>20$ & $>20$ \\
\hline 21 & E. faecium 4067/2014 & $\operatorname{van} A$ & $>96$ & 64 & $>20$ & $>20$ & $>20$ & $>20$ & $>20$ & $>20$ & $>20$ & $>20$ & $>20$ \\
\hline 22 & E. faecium 4167/2014 & $\operatorname{van} A$ & $>96$ & 64 & $>20$ & $>20$ & $>20$ & $>20$ & $>20$ & $>20$ & $>20$ & $>20$ & $>20$ \\
\hline 23 & E. faecium 4235/2014 & $\operatorname{van} A$ & $>96$ & 24 & $>20$ & 5 & 10 & 5 & $>20$ & $>20$ & 2.5 & 0.3125 & $>20$ \\
\hline 24 & E. faecium $5321 / 2014$ & $\operatorname{van} A$ & $>96$ & 24 & $>20$ & $>20$ & $>20$ & $>20$ & $>20$ & $>20$ & $>20$ & $>20$ & $>20$ \\
\hline 25 & E. faecium $5674 / 2014$ & $\operatorname{van} A$ & $>96$ & 24 & $>20$ & $>20$ & $>20$ & $>20$ & $>20$ & $>20$ & $>20$ & $>20$ & $>20$ \\
\hline 26 & E. faecium 6100/2014 & $\operatorname{van} A$ & $>96$ & 24 & $>20$ & $>20$ & $>20$ & $>20$ & 10 & 5 & 2.5 & 0.625 & $>20$ \\
\hline 27 & E. faecium $6421 / 2014$ & $\operatorname{van} A$ & $>96$ & 96 & $>20$ & $>20$ & $>20$ & $>20$ & $>20$ & $>20$ & $>20$ & $>20$ & $>20$ \\
\hline 28 & E. faecium $6823 / 2014$ & $\operatorname{van} A$ & $>96$ & 16 & $>20$ & $>20$ & $>20$ & $>20$ & $>20$ & $>20$ & $>20$ & $>20$ & $>20$ \\
\hline 29 & E. faecium 7009/2014 & $\operatorname{van} A$ & $>96$ & 64 & $>20$ & $>20$ & $>20$ & $>20$ & $>20$ & $>20$ & $>20$ & $>20$ & $>20$ \\
\hline 30 & E. faecium $8012 / 2014$ & $\operatorname{van} A$ & $>96$ & 96 & $>20$ & $>20$ & $>20$ & $>20$ & $>20$ & $>20$ & $>20$ & $>20$ & $>20$ \\
\hline 31 & E. faecalis $8046 / 2014$ & $\operatorname{van} A$ & $>96$ & 64 & 20 & 10 & 5 & 10 & 5 & 2.5 & 2.5 & 0.3125 & 20 \\
\hline 32 & E. faecium $8122 / 2014$ & $\operatorname{van} A$ & $>96$ & 64 & $>20$ & $>20$ & $>20$ & $>20$ & $>20$ & $>20$ & $>20$ & $>20$ & $>20$ \\
\hline 33 & E. faecium $8341 / 2014$ & $\operatorname{van} A$ & $>96$ & 64 & $>20$ & $>20$ & $>20$ & $>20$ & $>20$ & $>20$ & $>20$ & $>20$ & $>20$ \\
\hline 34 & E. faecium $8598 / 2014$ & $\operatorname{van} A$ & $>96$ & $>96$ & $>20$ & $>20$ & $>20$ & $>20$ & $>20$ & $>20$ & $>20$ & $>20$ & $>20$ \\
\hline 35 & E. faecalis $8673 / 2014$ & $\operatorname{van} A$ & $>96$ & $>96$ & $>20$ & 10 & 20 & 5 & 10 & $>20$ & 2.5 & 0.625 & $>20$ \\
\hline 36 & E. faecium 9065/2014 & $\operatorname{van} A$ & $>96$ & $>96$ & 20 & 20 & 10 & 5 & 5 & 20 & 5 & 1.25 & $>20$ \\
\hline 37 & E. faecium $9181 / 2014$ & $\operatorname{van} A$ & $>96$ & $>96$ & $>20$ & $>20$ & $>20$ & $>20$ & $>20$ & $>20$ & $>20$ & $>20$ & $>20$ \\
\hline 38 & E. faecium 9543/2014 & $\operatorname{van} A$ & $>96$ & $>96$ & $>20$ & $>20$ & $>20$ & $>20$ & $>20$ & $>20$ & $>20$ & $>20$ & $>20$ \\
\hline 39 & E. faecalis $9667 / 2014$ & $\operatorname{van} A$ & $>96$ & $>96$ & 0.3125 & 1.25 & 2.5 & 10 & 1.25 & $>20$ & 0.3125 & 0.625 & $>20$ \\
\hline 40 & E. faecium 9889/2014 & $\operatorname{van} A$ & $>96$ & $>96$ & 2.5 & 5 & 2.5 & 5 & 5 & 20 & 1.25 & 0.625 & 10 \\
\hline 41 & E. faecium 10008/2014 & $\operatorname{van} A$ & $>96$ & $>96$ & 5 & 20 & 2.5 & 5 & 20 & 20 & 2.5 & 1.25 & 20 \\
\hline
\end{tabular}

Abbreviation: MIC, minimum inhibitory concentration.

\section{Compound 20}

To a stirred solution of compound $19^{27}(107 \mathrm{mg}, 0.15 \mathrm{mmol})$ in $\mathrm{CH}_{2} \mathrm{Cl}_{2}$ $(30 \mathrm{ml})$, propargyl amine $(12 \mu \mathrm{l}, 0.19 \mathrm{mmol})$ and $\mathrm{Et}_{3} \mathrm{~N}(26 \mu \mathrm{l}, 0.19 \mathrm{mmol})$ were added under an argon atmosphere and stirred overnight at room temperature. The reaction mixture was diluted with $\mathrm{CH}_{2} \mathrm{Cl}_{2}(100 \mathrm{ml})$, washed with cc. aq $\mathrm{NH}_{4} \mathrm{Cl}(20 \mathrm{ml})$ and water $(2 \times 20 \mathrm{ml})$, dried and concentrated. The crude product was purified by flash column chromatography ( $n$-hexane:acetone 8:2) to give $20(102 \mathrm{mg}, 99 \%)$ as a yellow syrup. $[\alpha]_{\mathrm{D}}{ }^{25}=-87.5$ (c 0.11 , $\mathrm{CHCl}_{3}$ ); ${ }^{1} \mathrm{H}$ NMR $\left(360 \mathrm{MHz}, \mathrm{CDCl}_{3}\right) \delta$ (p.p.m.): 5.52 (d, $J=5.0 \mathrm{~Hz}, 2 \mathrm{H}$, $2 \mathrm{xH}-1), 4.70-4.52(\mathrm{~m}, 2 \mathrm{H}), 4.44-4.13(\mathrm{~m}, 4 \mathrm{H}), 4.06-3.85$ (m, $2 \mathrm{H}), 3.62-3.56$ (m, 2H), 3.46-3.30 (m, 2H), $2.17(\mathrm{~m}, 1 \mathrm{H}), 1.63-1.51(\mathrm{~m}, 2 \mathrm{H}), 1.46(\mathrm{~s}, 6 \mathrm{H})$, $1.44(\mathrm{~s}, 6 \mathrm{H}), 1.33(\mathrm{~s}, 6 \mathrm{H}), 1.32(\mathrm{~s}, 6 \mathrm{H}) .{ }^{13} \mathrm{C}$ NMR $\left(91 \mathrm{MHz}, \mathrm{CDCl}_{3}\right) \delta 165.3$ $(2 \times \mathrm{C}=\mathrm{O}), 127.6(\mathrm{C}=\mathrm{C}), 113.8$ (4 C, $\left.\mathrm{C}_{\text {acetalic }}\right), 96.6(2 \mathrm{C}-1), 71.6,71.0,70.6$, $68.0(2 \times \mathrm{C}-2,3,4,5), 31.7(2 \times \mathrm{C}-6), 26.0,26.0,24.9,24.5\left(8 \mathrm{C}, \mathrm{CH}_{3, \mathrm{ip}}\right)$.

\section{Compound 6}

To the solution of azido teicoplanin pseudoaglycon 18 (105 $\mathrm{mg}, 0.075 \mathrm{mmol})$ and the propargyl derivative $20(68 \mathrm{mg}, 0.09 \mathrm{mmol})$ in dry DMF $(5 \mathrm{ml}), \mathrm{CuI}$ $(1.4 \mathrm{mg}, 0.008 \mathrm{mmol})$ and $\mathrm{Et}_{3} \mathrm{~N}(10 \mu \mathrm{l}, 0.075 \mathrm{mmol})$ were added under an argon atmosphere and the mixture was stirred overnight. The reaction mixture 
Table 4 MIC of compounds 1-9 against VanA and VanB enterococci in $\mu \mathrm{g} \mathrm{ml}^{-1}$

\begin{tabular}{|c|c|c|c|c|c|c|c|c|c|c|c|c|c|}
\hline Number & Strain & $P C R$ & Vancomycin & Teicoplanin & 1 & 2 & 3 & 4 & 5 & 6 & 7 & 8 & 9 \\
\hline 42 & E. faecium 1333/2014 & $\operatorname{van} A, \operatorname{van} B$ & $>96$ & 12 & $>20$ & $>20$ & $>20$ & 10 & $>20$ & $>20$ & $>20$ & 1.25 & $>20$ \\
\hline 43 & E. faecium $6713 / 2014$ & $\operatorname{van} A, \operatorname{van} B$ & $>96$ & 32 & $>20$ & $>20$ & $>20$ & 5 & $>20$ & $>20$ & $>20$ & 5 & $>20$ \\
\hline 44 & E. faecium $9698 / 2014$ & $\operatorname{van} A, \operatorname{van} B$ & $>96$ & $>96$ & $>20$ & $>20$ & $>20$ & $>20$ & $>20$ & $>20$ & $>20$ & $>20$ & $>20$ \\
\hline
\end{tabular}

Abbreviation: MIC, minimum inhibitory concentration.

Table 5 Number of vancomycin- and teicoplanin-resistant Enterococcus strains susceptible to teicoplanin pseudoaglycon derivatives 1-9

\begin{tabular}{lccccccccc}
\hline Compound & $\mathbf{1}$ & $\mathbf{2}$ & $\mathbf{3}$ & $\mathbf{4}$ & $\mathbf{5}$ & $\mathbf{6}$ & $\mathbf{7}$ & $\mathbf{8}$ & $\mathbf{9}$ \\
\hline $\begin{array}{l}\text { Number of } \\
\text { susceptible } \\
\begin{array}{l}\text { Enterococcus } \\
\text { strains }\end{array}\end{array}$ & $5 / 36$ & $5 / 35$ & $9 / 36$ & $11 / 36$ & $6 / 36$ & $4 / 36$ & $11 / 36$ & $13 / 36$ & $2 / 36$ \\
\end{tabular}

was concentrated, and the crude product was purified by flash chromatography (toluene:MeOH 1:1+1\% acetic acid) and gel filtration by Sephadex-LH 20 gel in methanol to give $6(61 \mathrm{mg}, 41 \%)$ as a yellow powder. MALDI-TOF-MS m/z $2132.62(\mathrm{M}+\mathrm{Na})^{+}$; calcd. 2132.49 for $\mathrm{C}_{97} \mathrm{H}_{97} \mathrm{Cl}_{2} \mathrm{~N}_{11} \mathrm{NaO}_{35} \mathrm{~S}_{2}$.

\section{RESULTS AND DISCUSSION}

On the basis of the routine antibacterial screening (Supplementary information, Supplementary Table S1), teicoplanin pseudoaglycon derivatives 1-9 (Table 1) have been selected to the present antibacterial study. Synthesis of $\mathbf{3}^{27}$ and 7-9 has been published recently. ${ }^{28}$

Lipoic acid derivatives 1 and 2 were prepared from $\mathrm{N}$-acetylglucosaminyl teicoplanin aglycon ${ }^{29} \mathbf{1 0}$ by simple acylation reactions with hydroxysuccinimide active esters 11 and $12^{24}$ (Scheme 1).

In the framework of a systematic structure-anti-influenza virus activity study, ${ }^{30}$ compounds 4 and 5 have been prepared by derivatization of azido teicoplanin pseudoaglycon. The synthesis of the substituents 16 and 17 started from methyl 4,6-O-p-methoxybenzylidene- $\alpha$-D-glucopyranoside $13 .^{25}$ Di-O- $n$-butylation of 13 followed by reductive opening of the 4,6-acetal ring ${ }^{31,32}$ of the obtained 14 gave 15 . Subsequent alkylation of the liberated 6-hydroxyl group with a propargyl tetraethyleneglycol derivative produced 16, conjugation of which to azido teicoplanin pseudoaglycon $18^{20}$ by the copper catalyzed azide-alkyne dipolar cycloaddition click reaction $^{33,34}$ furnished compound 4. DDQ-mediated oxidative PMB deprotection of $\mathbf{1 6}$ resulted in $\mathbf{1 7}$. Then compound $\mathbf{5}$ was obtained using click reaction of $\mathbf{1 7}$ and $\mathbf{1 8}$ (Scheme 2).

In a search for antibacterial and antiviral teicoplanin pseudoaglycon derivatives, we have synthesized compound 6 by the copper catalyzed azide-alkyne click reaction of azide $\mathbf{1 8}$ with alkyne $\mathbf{2 0}$ obtained from $\mathrm{N}$-ethoxycarbonyl maleimide derivative $\mathbf{1 9}^{27}$ (Scheme 3).

Our derivatives were characterized using calculated $\log P$ values (Table 1). According to these calculations, eight of the nine compounds displayed $\log P$ values $>3$ at their isoelectric point, showing their high lipophilicity.

Evaluation of antibacterial activity on a standard panel of Grampositives showed that compounds 1-6 are very active against the two Enterococcus strains possessing resistance genes vanA or vanB. Compounds 7-9 that we have published earlier also displayed high activity against resistant enterococci (Supplementary Table S1).
In order to obtain structure-activity relationship information in this collection of teicoplanin pseudoaglycon derivatives, we evaluated the antibacterial activity of compounds 1-9 against a collection of 44 vancomycin-resistant Enterococcus faecalis and Enterococcus faecium strains isolated from routine laboratory samples of patients suffering from wound, urinary tract or blood stream infections. All the strains were characterized using PCR using primers for the identification of $v a n A$ - or vanB-resistance gene sequences. Eight of the strains proved to have vanB, 32 strains had vanA and interestingly 3 of them possessed both genes. ${ }^{35}$ The prevalence of VREs carrying the van $A$ gene is higher owing to the enhanced mobility of the mentioned gene cluster. ${ }^{36}$

Against the first group of enterococci (the VanB group, Table 2, lines 1-8), all of our nine compounds exhibited low MIC values, mostly lower than that of teicoplanin. In the evaluation of antibacterial activities against the vanA-positive group of E. faecalis and E. faecium (Table 3, lines 9-41) and for the group of three strains possessing both resistance genes (Table 4, lines 42-44), the same compounds displayed diverse results. Four of them $(3,4,7$ and 8$)$ proved to be active against about one-third of the Enterococcus strains; the other compounds had moderate or low activity. Analyzing the data further in Tables 3 and 4, it can be noticed that some of the strains are completely resistant to all of our teicoplanin pseudoaglycon derivatives, and some of them are more or less sensitive (see Table 5 for details). This phenomenon might be related to the different degrees of expression of the resistance genes in the different strains. ${ }^{37}$

The $\log P$ calculations for the whole collection of compounds revealed that all of them are lipophilic compounds with $\log P$ values close to or $>3$. Based on the results presented here and in our previous articles, ${ }^{16-21,27,28}$ it seems that there is no linear correlation between the lipophilicity of glycopeptide antibiotics and their antibacterial activity against VRE strains, but the presence of a hydrophobic moiety on the antibiotic is critical in most cases.

It has been shown that other attributes than lipophilicity can also substantially influence the activity of glycopeptide derivatives; for example, the overall charge ${ }^{38}$ and the capability of the compounds to dimerize ${ }^{39}$ in the presence of their target, D-Ala-D-Ala, and obviously the structure of the sidechain itself. As all molecules presented here are of acidic character, they are uniformly negatively charged at the $\mathrm{pH}$ of the antibacterial tests (close to neutral $\mathrm{pH}$ ). As the differences in activity owing to ionization characteristics can be ruled out and the dimerization of teicoplanin, its aglycon or pseudoaglycons was also shown to be insignificant, ${ }^{40}$ we can conclude that among these derivatives the structure of the substituents is the most important factor in their activity against VRE. Considering these and some of our other results (Szucs Z, unpublished data) relatively simple aromatic substituents like phenyl, biphenyl, naphthyl, etc. seem to be very beneficial for high in vitro antibacterial activity.

In summary, evaluation of antibacterial activities of nine teicoplanin pseudoaglycon derivatives against a series of resistant enterococci demonstrated that introduction of hydrophobic side chains into the antibiotic molecule, in spite of the possible side effects of this structure 
manipulation, is still a good strategy in fighting against bacterial resistance. Compounds 4, 7 and 8 could be good lead derivatives for obtaining new highly active antibiotics against multiresistant enterococci.

\section{CONFLICT OF INTEREST}

The authors declare no conflict of interest.

1 Rex, J. H. ND4BB: addressing the antimicrobial resistance crisis. Nat. Rev. Microbiol. 12, 231-232 (2014).

2 Eichberg, M. J. Public funding of clinical-stage antibiotic development in the United States and European Union. Health Security 13, 156-165 (2015).

3 World Health Organization. Antimicrobial Resistance: Global Report on Surveillance, (WHO Press, Geneva, Switzerland, 2014).

4 Centers for Disease Control and Prevention. Antibiotic resistance threats in the United States, 2013. Available at http://www.cdc.gov/drugresistance/pdf/ar-threats-2013-508. pdf.

5 Cooper, R. D. et al. Reductive alkylation of glycopeptide antibiotics: synthesis and antibacterial activity. J. Antibiot. 49, 575-581 (1996).

6 Thompson, R. C., Wilkie, S. C. \& Zweifel, M. J. (Eli Lilly \& Co.) Selective n-Acylation of a82846 Glycopeptide Analogs W02001058933A3 (2001).

7 Malabarba, A. et al. $\mathrm{N}^{15}$-alkyl and $\mathrm{N}^{15}, \mathrm{~N}^{15}$-dialkyl derivatives of teicoplanin antibiotics. J. Antibiot. 43, 1107-1121 (1990).

8 Malabarba, A. et al. $\mathrm{N}^{63}$-carboxamides of $\mathrm{N}^{15}$-alkyl and $\mathrm{N}^{15}, \mathrm{~N}^{15}$-dialkyl derivatives of teicoplanin and deglucoteicoplanin. J. Antibiot. 46, 668-675 (1993).

9 Pavlov, A. Y. et al. Carboxamides and hydrazide of glycopeptide antibiotic eremomycin. Synthesis and antibacterial activity. J. Antibiot. 49, 194-198 (1996).

10 Pavlov, A. Y., Lazhko, E. I. \& Preobrazhenskaya, M. N. A new type of chemical modification of glycopeptides antibiotics: aminomethylated derivatives of eremomycin and their antibacterial activity. J. Antibiot. 50, 509-513 (1997).

11 Preobrazhenskaya, M. N. et al. N'-( $\alpha$-aminoacyl)- and $\mathrm{N}^{\prime}-\alpha-\left(\mathrm{N}^{\alpha}\right.$-alkylamino)acyl derivatives of vancomycin and eremomycin. J. Antibiot. 60, 235-244 (2007).

12 Crane, C. M. et al. Synthesis and evaluation of selected key methyl ether derivatives of vancomycin aglycon. J. Med. Chem. 53, 7229-7235 (2010).

13 Leadbetter, M. R. et al. Hydrophobic vancomycin derivatives with improved ADME properties: discovery of telavancin (TD-6424). J. Antibiot. 57, 326-336 (2004).

14 Malabarba, A. \& Goldstein, B. P. Origin, structure, and activity in vitro and in vivo of dalbavancin. J. Antimicrob. Chemother. 55(Suppl. S2): ii15-ii20 (2005).

15 Fraise, A. P. \& Andrews, J. Wise, R. Activity of a new glycopeptide antibiotic (LY333328) against enterococci and other resistant Gram-positive organisms. J. Antimicrob. Chemother. 40, 423-425 (1997).

16 Sztaricskai, F. et al. A new series of glycopeptide antibiotics incorporating a squaric acid moiety. J. Antibiot. 59, 564-582 (2006).

17 Sipos, A. et al. Synthesis of isoindole and benzoisoindole derivatives of teicoplanin pseudoaglycon with remarkable antibacterial and antiviral activities. Bioorg. Med. Chem. Lett. 22, $7092-7096$ (2012).

18 Sipos, A. et al. Synthesis of fluorescent ristocetin aglycon derivatives with remarkable antibacterial and antiviral activities. Eur. J. Med. Chem. 58, 361-367 (2012).

19 Bereczki, I. et al. A few atoms make the difference: synthetic, CD, NMR and computational studies on antiviral and antibacterial activities of glycopeptide antibiotic aglycon derivatives. Eur. J. Med. Chem. 94, 73-86 (2015).
20 Pintér, G. et al. Diazo transfer - Click reaction route to new, lipophilic teicoplanin and ristocetin aglycon derivatives with high antibacterial and anti-influenza virus activity: an aggregation and receptor binding study. J. Med. Chem. 52, 6053-6061 (2009).

21 Tollas, Sz. et al. Nano-sized clusters of a teicoplanin $\psi$-aglycon-fullerene conjugate Synthesis, antibacterial activity and aggregation studies. Eur. J. Med. Chem. 54, 943-948 (2012).

22 The European Committee on Antimicrobial Susceptibility Testing. Breakpoint tables for interpretation of MICs and zone diameters. Version 4.0 (2014). Available at http://www. eucast.org/fileadmin/src/media/PDFs/EUCAST_files/Breakpoint_tables/Breakpoint_table v 4.0.xls.

23 Dutka-Malen, S., Evers, S. \& Courvalin, P. Detection of glycopeptides resistance genotypes and identification to the species level of clinically relevant enterococci by PCR. J. Clin. Microbiol. 33, 24-27 (1995).

$24 \mathrm{Li}$, J. J. \& Algar, W. R. A long-wavelength quantum dot-concentric FRET configuration: characterization and application in a multiplexed hybridization assay. Analyst 141, 3636-3647 (2016)

25 Moitessier, N., Englebiennea, P. \& Chapleur, Y. Directing-protecting groups for carbohydrates. Design, conformational study, synthesis and application to regioselective functionalization. Tetrahedron 61, 6839-6853 (2005).

26 Pintér, G. et al. The effect of systematic structural modifications on the antibacterial activity of novel oxazolidinones. Med. Chem. 7, 45-55 (2011).

27 Csávás, M. et al. Synthesis and antibacterial evaluation of some teicoplanin pseudoaglycon derivatives containing alkyl- and arylthiosubstituted maleimides. J. Antibiot. 68, 579-585 (2015).

28 Szűcs, Z. et al. Synthesis and biological evaluation of lipophilic teicoplanin pseudoaglycon derivatives containing a substituted triazole function. J. Antibiot. 70, 152-157 (2017).

29 Malabarba, A. et al. Teicoplanin, antibiotics from Actinoplanes teichomyceticus nov. sp. VI. Chemical degradation: physico-chemical and biological properties of acid hydrolysis products. J. Antibiot. 37, 988-999 (1984).

30 Bereczki, I. et al. Semisynthetic teicoplanin derivatives as new influenza virus binding inhibitors: synthesis and antiviral studies. Bioorg. Med. Chem. Lett. 24, 3251-3254 (2014).

31 Herczeg, M. et al. Novel syntheses of Idraparinux, the anticoagulant pentasaccharide with indirect selective factor Xa inhibitory activity. Tetrahedron 69, 3149-3158 (2013).

32 Herczeg, M., Lázár, L., Ohlin, M., Borbás, A. in Carbohydrate Chemistry: Proven Synthetic Methods Vol. 2 (eds van der Marel, G. \& Codée, J. 7-18 (CRC Press, Taylor \& Francis Group, Boca Raton, USA, 2014).

33 Rostovtsev, V. V., Green, L. G., Fokin, V. V. \& Sharpless, K. B. A stepwise Huisgen cycloaddition process: copper(I)-catalyzed regioselective 'ligation' of azides and terminal alkynes. Angew. Chem. Int. Ed. 41, 2596-2599 (2002).

34 Tornøe, C. W., Christensen, C. \& Meldal, M. Peptidotriazoles on solid phase: $[1,2,3]$-triazoles by regiospecific copper(I)-catalyzed 1,3-dipolar cycloadditions of terminal alkynes to azides. J. Org. Chem. 67, 3057-3064 (2002).

35 Gousia, P., Economou, V., Bozidis, P. \& Papadopoulou, C. Vancomycin-resistance phenotypes, vancomycin-resistance genes, and resistance to antibiotics of enterococci isolated from food of animal origin. Foodborne Pathog. Dis. 12, 214-220 (2015)

36 Corso, A. C. et al. Molecular epidemiology of vancomycin-resistant Enterococcus faecium in Argentina. Int. J. Infect. Dis. 11, 69-75 (2007).

37 Arthur, M., Depardieu, F., Reynolds, P. \& Courvalin, P. Moderate-level resistance to glycopeptide LY333328 mediated by genes of the vanA and vanB clusters in Enterococci. Antimicrob. Agents Chemother. 43, 1875-1880 (1999).

38 Malabarba, A., Nicas, T. I. \& Thompson, R. C. Structural modifications of glycopeptide antibiotics. Med. Res. Rev. 17, 69-137 (1997).

39 Allen, N. E. \& Nicas, T. E. Mechanism of action of oritavancin and related glycopeptide antibiotics. FEMS Microbiol. Rev. 26, 511-532 (2003).

40 Mackay, J. P. et al. Dissection of the contributions toward dimerization of glycopeptide antibiotics. J. Am. Chem. Soc 116, 4573-4580 (1994).

Supplementary Information accompanies the paper on The Journal of Antibiotics website (http://www.nature.com/ja) 